\title{
On the nonparaxial corrections of Bessel-Gauss beams
}

\author{
Omar El Gawhary ${ }^{1, *}$ and Sergio Severini ${ }^{2}$ \\ ${ }^{1}$ Optics Research Group, Department of Imaging Science and Technology, Delft University of Technology, \\ P.O. Box 5046, 2600 GA Delft, The Netherlands \\ ${ }^{2}$ Centro Interforze Studi per le Applicazioni Militari, Via della Bigattiera 10, 56122 San Piero a Grado (Pi), Italy \\ *Corresponding author: o.elgawhary@tudelft.nl
}

Received November 20, 2009; revised January 8, 2010; accepted January 13, 2010; posted January 13, 2010 (Doc. ID 120201); published February 23, 2010

\begin{abstract}
The nonparaxial corrections for Bessel-Gauss beams were derived recently using two different approaches [Borghi et al., J. Opt. Soc. Am. A 18, 1618 (2001) and Vaveliuk et al., J. Opt. Soc. Am. A 24, 3297 (2007)]. However, the two obtained results do not agree, so it is necessary to determine which method is correct. In the most recent of those papers, Vaveliuk et al. claimed that their method is correct while the method described by Borghi et al. is incorrect. In the present work, just by solving the rigorous propagation problem, we show that exactly the converse is true. (C) 2010 Optical Society of America
\end{abstract}

OCIS codes: $030.4070,260.1960,350.5500$.

\section{INTRODUCTION}

The classical propagation problem in optics deals with the computation of a field distribution in a half-space once the field value (or its derivative) on the input plane, located at $z=0$, say, is known. Within the scalar theory framework, if we denote the field as $U(x, y, z)$, that implies looking for a solution of the scalar Helmholtz equation

$$
\nabla^{2} U(x, y, z)+k^{2} U(x, y, z)=0
$$

( $k=2 \pi / \lambda, \lambda$ being the wavelength), with the assigned given boundary conditions (Dirichlet problem)

$$
\left.U(x, y, z)\right|_{z=0}=U(x, y, 0)
$$

in the domain $\{x, y, z>0\}$. Generally speaking, it is rare to get a closed-form expression for the solution of this problem; therefore some approximations became quite popular within the scientific community. Surely the most important is the paraxial or Fresnel approximation, which is valid, as is well known, when the field is a plane wave modulated by a slowly varying envelope,

$$
U(x, y, z)=V(x, y, z) \exp (i k z),
$$

where the envelope $V(x, y, z)$ is the solution of the socalled paraxial wave equation

$$
\nabla_{\perp}^{2} V(x, y, z)+2 i k \partial_{z} V(x, y, z)=0,
$$

where $\nabla_{\perp}^{2}=\partial_{x}^{2}+\partial_{y}^{2}$. Equation (4) is probably so important because of its ability to describe the radiation emitted by many laser sources. Usually finding a solution of the paraxial problem is easier than the original problem; thus recently many studies have tried to build a nonparaxial solution, starting from the paraxial approximation, by adding to it higher-order nonparaxial corrections. Here we will focus on two of those methods, because they do not lead to the same conclusions and therefore it must be determined which of them is correct and which is not. The first method [1] (which we call the BSP method) was introduced by Borghi, Santarsiero, and Porras in 2001 and makes use of the perturbative technique introduced by Lax et al. [2] in combination with a technique described by Wünsche [3], which uses specific differential operators to generate all nonparaxial corrections of a given initial paraxial solution. This method was applied in [1] to the interesting case of Bessel-Gauss beams, and the closedform expression for the nonparaxial corrections of any order were given. The issue of finding all nonparaxial corrections in the case of Bessel-Gauss beams was faced, more recently, also by Vaveliuk et al. in another work [4]. In this different approach (which we indicate as the VZMR method) the nonparaxial corrections were obtained by combining the propagation operator method with the so-called $p$-index Fourier transform.

In their paper Vaveliuk et al. emphasized the differences between the results provided by the two different works and used an indirect method to understand which was the correct one. They considered the case of a zeroorder Bessel-Gauss distribution on $z=0$, of the type

$$
J_{0}\left(\beta r_{\perp}\right) \exp \left(-r_{\perp}^{2} / w_{0}^{2}\right)
$$

where $r_{\perp}=\sqrt{x^{2}+y^{2}}, w_{0}$ is the waist of the Gaussian term, and $\beta$ is the spatial frequency characterizing the Bessel field. In particular, in Fig. 1 of their paper [4], the paraxial solution, along with the first two nonparaxial corrections on the $z$ axis, obtained through the two different approaches, were plotted. In that plot the field parameters $\beta$ and $w_{0}$ were chosen such that $k w_{0}=200$ and $\beta w_{0}$ $=60$.

The problem is that the nonparaxial corrections obtained through the two methods are quite different. More importantly, while in the VZMR method they are really small, which means that the paraxial model is accurate enough to describe the field under study, in the BSP 


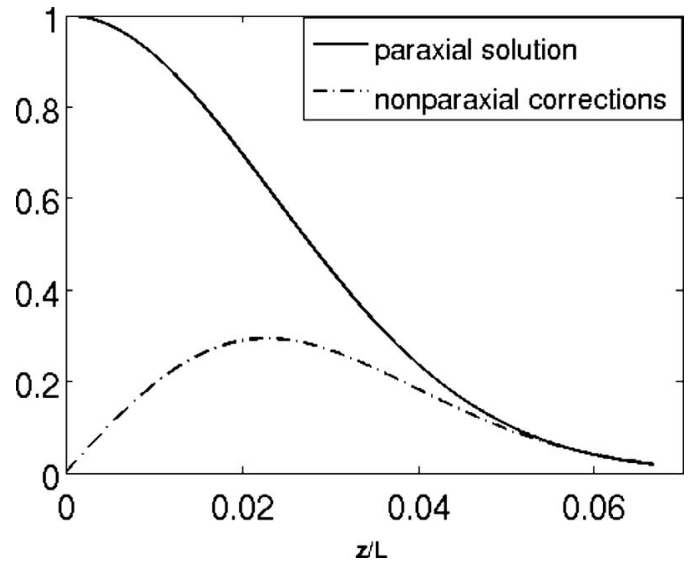

Fig. 1. Field modulus plotted on the $z$ axis as a function of the dimensionless variable $z / L$ : paraxial approximation (solid curve) and nonparaxial corrections (dotted curve) for a Bessel-Gauss input field distribution of the type $\exp \left(-r_{\perp}^{2} / w_{0}^{2}\right) J_{0}\left(\beta r_{\perp}\right) . w_{0} / \lambda$ $=200 /(2 \pi)$ and $\beta w_{0}=60$ as in [4], Fig. 1. $L=\pi w_{0}^{2} / \lambda$ is the Rayleigh distance. The nonparaxial corrections are evaluated through the Rayleigh-Sommerfeld formula of the first kind.

method they are not negligible at all. That means that for Borghi et al. the field is not paraxial and the paraxial model provides a rough estimate of the actual field. Even if the two schemes are different, so that it is not correct to compare directly the corresponding corrections, the authors are led to two completely different conclusions for the same field: for Vaveliuk et al. the field is paraxial, while for Borghi et al. the field cannot be considered paraxial at all. In our opinion that is a serious issue that must be investigated in order to avoid theory misinterpretations and, in addition, erroneous conclusions in future works potentially based on these models. In the next section we face that issue, and we show which of the two approaches, in our opinion, has to be considered correct.

\section{CHECK BASED ON RIGOROUS PROPAGATION INTEGRALS}

As we said before, it is important to understand which method is correct. Of course, since one wants to validate a theory, it is necessary to have recourse to an independent method to do that. Apart from being independent, it has to be rigorous and universally recognized as correct. Fortunately, such a method exists. In fact it is known that an exact solution of the Helmholtz equation, with Dirichlet boundary conditions, is provided by the RayleighSommerfeld propagation integral,

$$
U(x, y, z)=-\frac{1}{2 \pi} \iint U(\xi, \eta, 0) \partial_{z}\left[\frac{\exp (i k r)}{r}\right] d \xi d \eta,
$$

where $r=\sqrt{(x-\xi)^{2}+(y-\eta)^{2}+z^{2}}$ and $U(\xi, \eta, 0)$ is the field on the input plane $(z=0)$. Equation $(6)$ is a rigorous solution of the Helmholtz equation that can be found in every standard book about optics and electromagnetism [5-7]. From it derives the analytical formulation of the Huygens-Fresnel principle as well as the standard Fresnel and Fraunhofer approximations.

Thus, the simple thing to do is to propagate the input distribution in Eq. (5) by using formula (6). To evaluate the weight of the nonparaxial corrections, one also needs the paraxial solution, which for a zero-order BesselGauss beam is known in closed form and is given as [8-10]

$$
\begin{aligned}
U_{\text {parax }}(x, y, z) & \\
= & {\left[\frac{1}{1+i z / L}\right] J_{0}\left[\frac{\beta r_{\perp}}{(1+i z / L)}\right] \exp \left[-\frac{r_{\perp}^{2}}{w_{0}^{2}(1+i z / L)}\right] } \\
& \times \exp \left[-i \frac{\beta^{2} z}{2 k(1+i z / L)}\right] \exp (i k z),
\end{aligned}
$$

where $r_{\perp}=\sqrt{x^{2}+y^{2}}, L=\pi w_{0}^{2} / \lambda$ is the Rayleigh distance.

We can always write the exact solution as

$$
U(x, y, z)=U_{\text {parax }}(x, y, z)+\Delta U(x, y, z)
$$

,where $\Delta U(x, y, z)=U(x, y, z)-U_{\text {parax }}(x, y, z)$ represents the nonparaxial corrections. After solving the RayleighSommerfeld formula we get $U(x, y, z)$ so that we can plot the paraxial solution, together with $\Delta U(x, y, z)$, to understand whether the nonparaxial corrections are negligible. Because the correctness of Eq. (6) is not debatable, that represents a conclusive check: only the method that is in agreement with it can be considered correct, while the other one must be discarded. The result is plotted in Fig. 1 when the same field $\left(k w_{0}=200, \beta w_{0}=60\right)$ as that in Fig. 1 of [4] is considered. It is manifest that the nonparaxial corrections, reaching almost $30 \%$ of the maximum paraxial amplitude, are not negligible, so the field is not paraxial. Furthermore, the nonparaxial corrections (of which the first two are, of course, the most important ones) obtained through the BSP method (Fig. $2 c$ of [1] or Fig. 1(a) of [4]) fully agree, in behavior and strength, with the exact corrections derived in the present work. In contrast, the VZMR method seriously underestimates them (Fig. 1(b) of [4]). Since the first two corrections are supposed to be the dominant ones, and in [4] they are so small, Vaveliuk et al. were induced to believe, wrongly, that the field was paraxial.

But how did these authors conclude that their theory was correct then? Actually, they did not check the consistency of the model with the rigorous propagation formula, as we have done here, but they made an indirect analysis using a parameter recently introduced by some of them [11]. The problem with that parameter, as was already pointed out elsewhere $[12,13]$, is that it has a real physical meaning only within the paraxial regime. Outside that framework, it is completely meaningless. So using it to analyze the paraxiality of nonparaxial fields, like the case we are talking about here, is simply misleading. Stated differently, that parameter said that the field was paraxial, while, as we have just proved here, the nonparaxial corrections were not negligible at all. It follows that one cannot rely on that parameter to determine whether a field is paraxial or not.

Still, one has to understand how the VZMR method produced the wrong corrections. Actually, in their paper the authors make a really puzzling assertion about evanescent waves that, if true, can be at the origin of the problem. 
They wrote that by using the $p$-index Fourier transform they do not need to postulate the existence of evanescent waves for fields propagating in free space. In fact, on properly choosing the $p$ parameter, they could act in order to have, basically, only homogeneous waves in the field spectrum. So apparently they can remove the existence of evanescent waves by just redefining, properly, the Fourier transform. But, if true, it would not be difficult to prove that such an assertion, alone, would imply the possibility of realizing a perfect imaging in free space. In fact, it is well known, theoretically and experimentally, that the nonpropagating nature of evanescent waves is the reason that the resolution limit exists. Without that, there would be no limitation in imaging details much smaller than $\lambda / 2$, measuring only the far field produced by a subwavelength source. No Rayleigh limit would exist. In that case the entire body of research in the field of metamaterials, surface plasmons, multiple scattering, and so on, to overcome that limit would not be justified [14-16]. In fact, it would simply suffice to properly redefine the Fourier transform. Needless to say, the scenario is a bit more complicated than that [17].

\section{CONCLUSIONS}

To summarize, we have proved that the nonparaxial corrections obtained by Borghi et al. [1] fully agree with the exact nonparaxial corrections as derived using the rigorous Rayleigh-Sommerfeld diffraction formula of the first kind (Dirichlet boundary conditions). In contrast, the method introduced by Vaveliuk et al. in [4] suffers from problems and does not provide the right estimations for those corrections. Also, the present work has proved how misleading the use of the parameter introduced in [11] to analyze the paraxiality of a field of interest can be. That parameter should not be used to check whether a field is paraxial, because it leads to strong misinterpretations of theory.

\section{ACKNOWLEDGMENT}

The authors are grateful to Massimo Santarsiero for useful discussions.

\section{REFERENCES}

1. R. Borghi, S. Santarsiero, and M. A. Porras, "Nonparaxial Bessel-Gauss beams," J. Opt. Soc. Am. A 18, 1618-1625 (2001).

2. M. Lax, W. H. Louisell, and W. B. McKnight, "From Maxwell to paraxial wave optics," Phys. Rev. A 11, 1365-1370 (1975)

3. A. Wünsche, "Transition from the paraxial approximation to exact solutions of the wave equation and application to Gaussian beams," J. Opt. Soc. Am. A 9, 765-774 (1992).

4. P. Vaveliuk, G. F. Zebende, M. A. Moret, and B. Ruiz, "Propagating free-space nonparaxial beams," J. Opt. Soc. Am. A 24, 3297-3302 (2007).

5. M. Born and E. Wolf, Principles of Optics, 7th (expanded) ed. (Cambridge University Press, 2001), p. 514.

6. R. K. Luneburg, Mathematical Theory of Optics (University of California Press, 1964), p. 316.

7. M. Nieto-Vesperinas, Scattering and Diffraction in Physical Optics, 2nd ed. (World Scientific 2006), p. 179.

8. C. J. R. Sheppard and T. Wilson, "Gaussian-beam theory of lenses with annular aperture," IEE J. Microwaves, Opt. Acoust. 2, 105-112 (1978).

9. F. Gori, G. Guattari, and C. Padovani, "Bessel-Gauss beams," Opt. Commun. 64, 491-495 (1987).

10. O. El Gawhary and S. Severini, "Lorentz beams and symmetry properties in paraxial optics," J. Opt A: Pure Appl. Opt. 8, 409-414 (2006).

11. P. Vaveliuk, B. Ruiz, and A. Lencina, "Limits of the paraxial approximation in laser beams," Opt. Lett. 32, 927-929 (2007).

12. O. El Gawhary and S. Severini, "Reply to comment on degree of paraxiality for monochromatic light beams," Opt. Lett. 33, 3006 (2008).

13. O. El Gawhary and S. Severini, "Degree of paraxiality for monochromatic light beams," Opt. Lett. 33, 1360-1362 (2008).

14. J. B. Pendry, "Negative refraction makes a perfect lens," Phys. Rev. Lett. 18, 3966-3969 (2000).

15. S. Kawata, T. Ono, and P. Verma, "Subwavelength colour imaging with a metallic nanolens," Nature Photon. 2, 438-442 (2008).

16. F. Simonetti, "Multiple scattering: the key to unravel the subwavelength world from the far-field pattern of a scattered wave," Phys. Rev. E 73, 036619 (13pp.) (2006).

17. C. J. R. Sheppard, M. A. Alonso, M. Santarsiero, and R. Borghi, "Comment on 'Do evanescent waves really exist in free space?"' Opt. Commun. 266, 448-449 (2006). 